\title{
WOUND MAGNETOSTATICS AND FLARING
}

\author{
C.M. BOILY \\ I.U.C.A.A., Post Bag 4, \\ Ganeshkhind, Pune 411 007, India \\ and \\ D. LYNDEN-BELL \\ Institute of Astronomy, Madingley Road, Cambridge, CB3 OHA
}

\begin{abstract}
We discuss the winding of a force-free axisymmetric magnetic field rooted on a heavy conductor on $z=0$. In quadrupolar symmetry the field expands in the half-space $z>0$ and the toroidal flux concentrates on a conical surface. After a mean twist of $208^{\circ}$, the conical layer hosts large toroidal current loops with reversal of the magnetic flux on either side. The evolution of the field structure is described by scale-free static solutions $B \sim r^{-(p+2)}$, with $p$ taking values between 0 and 2 . The large expansion factor of the field structure is suggestive of flaring originating on the solar photosphere.
\end{abstract}

Key words: mhd - Solar flares

\section{Problematic}

Shearing motions in the highly conducting photosphere drag the foot-points of emerging field lines. As a result mechanical energy is invested in the force-free field in the atmosphere above. There the dilute plasma freezes around the lines of force. We picture the photosphere as an infinite, heavy conducting sheet on $z=0$ where a discus of diameter $2 a$ located at the centre rotates about the z-axis giving rise to shear. Let the (axisymmetric) magnetic flux $\Phi$ emerging through $\pi a^{2}$ reconnect on $z=0$ in the region $R>a$. The foot-points of the field are rooted on $z=0$ so what happens now that the disc rotates? For slow rotation the field assumes static equilibrium configurations. Provided only the field vanishes high above $z=0$, the field components will satisfy (Aly 1984; Lynden-Bell 1994)

$$
\int_{V} B_{z}^{2} \mathrm{~d} V=\int_{V} B_{R}^{2}+B_{\theta}^{2} \mathrm{~d} V
$$

Since $B_{R}$ and $B_{z}$ are fixed on $z=0$ they cannot increase there, however as the surface $\pi a^{2}$ is rotated the toroidal flux increases. This suggests that in order to satisfy the above integral the whole structure expands in $R$ and $z$ with flux-freezing. On $z=0$ there can be no expansion so after some twist the field structure becomes approximately of quadrupolar symmetry, while the length $a$ brings no useful unit of measure. We therefore look for scale-free axisymmetric solutions to the problem with four-fold symmetry. 


\section{Equations}

We follow Lynden-Bell \& Boily (1994) with a slight change of notation. Since $B-V \nabla \cdot B-V B=0$ we write the axisymmetric $B-V B$

$$
B-V B=B-V \nabla \phi \times B-V \nabla \Psi+R B_{\phi} B-V \nabla \phi=B-V B_{\perp}+B_{\phi} \hat{\phi},
$$

where $\phi$ is the azimuth and both $\Psi$ and $B_{\phi}$ are functions of the cylindrical radius $R$ and $z . \Psi$ is taken to be

$$
\Psi(r, \theta) \equiv a^{p} \Phi r^{-p} f(\mu)
$$

with $f(\mu)$ a dimensionless function of $\mu \equiv \cos \theta$ and $p$ a free parameter. For a force-free field we may write $B-V \nabla \times B-V B=\alpha B-V B$, with $\alpha=\alpha(R, z)$. In spherical polar coordinates $f$ must satisfy

$$
\left[p(p+1) f+\left(1-\mu^{2}\right) f^{\prime \prime}\right] f^{-(p+2) / p}=-\left(a^{p} \Phi\right)^{\frac{2}{p}} \Psi^{-\frac{(p+2)}{p}} \frac{1}{2} \frac{\mathrm{d}\left(R B_{\phi}\right)^{2}}{\mathrm{~d} \Psi} .
$$

Since the left-hand side is a function of $\mu$ alone and the right-hand side is a function of $\Psi(R, z)$ we equate both to a dimensionless constant $(\equiv-C)$ and obtain two equalities:

$$
\begin{aligned}
& p(p+1) f+\left(1-\mu^{2}\right) f^{\prime \prime}=-C f^{(p+2) / p}, \\
& \frac{1}{2}\left(a^{p} \Phi\right)^{2 / p} \frac{\mathrm{d}\left(R B_{\phi}\right)^{2}}{\mathrm{~d} \Psi}=+C \Psi^{(p+2) / p} .
\end{aligned}
$$

Eq. (5) is integrated subject to $B_{\phi} \rightarrow 0$ when $r \rightarrow \infty$ so $B_{\phi} \rightarrow B_{\phi}(\Psi)$ is known where $\Psi$ is given by (2) with $f$ the solution of (4).

Because $f$ is an angular function, we modify the shape of the boundary into a half-sphere of radius $a$. Now all the flux emerges through the cap $\mu_{1} \leq \mu \leq 1$, with $\mu_{1}$ an unknown angle. Since the field decays rapidly with $R$ the bulk of the flux will reconnect in the vicinity of $R=a$. No length will appear in the boundary condition if we impose that all lines reconnect at the base of the sphere, on $0 \leq \mu \leq \mu_{1}$. Thus outside $r=a$ the flux through $z=0$ vanishes, giving the boundary condition $B_{\theta}=f=0$ when $\mu=0$. Since $B_{\phi}=0$ down the pole we require $\Psi=f=0$ where $\mu=1$.

The function $f(\mu)$ maximises at $\mu_{1}$; we use our freedom in $C$ so that $f\left(\mu_{1}\right)=1$. In terms of the old flux $\Phi$, the flux through the spherical cap at $r=a$ is $\Phi^{\prime}=2 \pi \Phi$. Our task consists in solving for $f$ in the differential (4) and discover the set of eigenvalues $C(p)$ with $p$ decreasing from 2 to 0 . The field components in $r \geq a$ are given by

$$
B-V B=\frac{a^{p} \Phi}{r^{p+2}}\left[\frac{-p f}{\sqrt{1-\mu^{2}}} \hat{\theta}+f^{\prime} \hat{r}+\frac{\sqrt{p C /(p+1)}}{\sqrt{1-\mu^{2}}} f^{1+1 / p} \hat{\phi}\right] .
$$




\section{Quadrupolar solutions}

Initially the quadrupolar field is meridional so $B_{\phi}=C=0$ with $p=2$. For these values of $C$ and $p,(4)$ admits a unique solution satisfying all boundary conditions, $f(\mu)=\frac{3 \sqrt{3}}{2} \mu\left(1-\mu^{2}\right)$ which maximises at $\mu_{1}=1 / \sqrt{3}$ where $f=1$. The solution for $B-V B$ follows from substituting for $f$ in (6). Solutions with $p<2$ have $C \neq 0$ and give rise to toroidal flux $B_{\phi}$. What is the corresponding number of turns? Because the field lines are rooted on $r=a$, each revolution of the cap $\mu_{1}<\mu<1$ with respect to the base $\mu<\mu_{1}$ increases the toroidal flux by one unit of $\Phi^{\prime}$. Thus we define the flux-weighted mean twist $N$ as the ratio of toroidal to meridional fluxes,

$$
N \equiv \frac{\Phi_{\phi}}{\Phi^{\prime}}=\frac{1}{2 \pi \Phi} \int_{0}^{\frac{\pi}{2}} \int_{a}^{\infty} B_{\phi} r \mathrm{~d} r \mathrm{~d} \theta=\left[\frac{C}{p(p+1)}\right]^{\frac{1}{2}} \int_{0}^{1} \frac{f^{(p+1) / p}}{1-\mu^{2}} \frac{\mathrm{d} \mu}{2 \pi} .
$$

From (7), an increase in $C$ conjointly with a decrease of $p$ contributes to augment $N$. We remark that in the original problem with foot-points rooted on the surface, all field lines would necessarily go through the same number of turn $N$. Here the boundary conditions do not forbid movements of the foot-points on $r=a$, which are recovered a posteriori from the solution $f$.

\subsection{Limiting SOLUTion: $p \rightarrow 0$}

When $p \rightarrow 0$ and since $\max \{f\}=1$, (4) reduces to $f^{\prime \prime}=0$ away from $\mu=\mu_{1}$, where $f=1$. Near the maximum we need solve $\left(1-\mu_{1}^{2}\right) f^{\prime \prime}=-C f^{(p+2) / p}$. The solution to this equation satisfying $f=0$ at $\mu=0$ or 1 is

$$
f(\mu)=1-\frac{p}{p+1} \ln \left[\cosh \left(\frac{C^{\prime}}{2}[1-2 \mu]\right)\right],
$$

and $f=1$ at $\mu_{1}=1 / 2$. In the above $\left(C^{\prime}\right)^{2}=4(p+1) C /(3 p)$. Substituting (8) in (7) we obtain a mean turn $N=1 /(p+1) \sqrt{3}$ and therefore $N=1 / \sqrt{3}$ when $p=0$. The field configuration is deduced from (6). As $p$ is reduced the field lines expand and become more radial (Fig. 1 of Lynden-Bell \& Boily 1994). With $p$ small the toroidal flux is tiny except where $f \simeq 1$, where $f$ is given by (8). Thus all the twist is confined to a conical surface at $\mu_{1} \rightarrow 1 / 2$, or $\theta \rightarrow 60^{\circ}$.

\subsection{Similarity Profile}

The solution with $p=0$ has the interesting property that all field lines turn by the same amount. The angle turned through $\mathrm{d} \phi$ expressed as function of $\mu=\cos \theta$ is

$$
\mathrm{d} \phi=\frac{C^{\prime} \sqrt{1-\mu_{1}^{2}}}{p+1} \frac{f^{1 / p}}{1-\mu^{2}} \mathrm{~d} \mu .
$$


For $p$ small the integrand is heavily weighted at $\mu=\mu_{1}$. Thus most of the twist $\mathrm{d} \phi$ occurs there. Since all field lines must go through $\mu=\mu_{1}$ this will be true for all of them. Integrating with the help of (8) we find $\phi=2 \pi N$ for $p=0$, that is, all field lines make $N$ turn, as in the original problem with foot-points rooted on $z=0$. This result does not hold for other values of $p$ since now the range $\mathrm{d} \mu$ for which $f^{1 / p}$ is appreciable depends on the latitudes of the foot-points on $r=a$, which by definition are different for different field lines.

\section{Summary}

Shear flows on the photosphere generate torques acting on an otherwise static field at equilibrium and cause it to expand by large factors. Thus the characteristic size of the shearing pattern is not important to the large-scale structure of the field. We idealised this situation and looked for scale-free power-law solutions to the magnetostatic equations in axisymmetry when flux lines emerge from a half-sphere of radius $a$. However in solving for the field structure the constraint that the foot-points be frozen on $r=a$ was relaxed. Thus in the initial configuration, the separatrix of outgoing and incoming flux lines is at $\mu_{1}=1 / \sqrt{3}$ or $54.7^{\circ}$, whereas in the final configuration the separatrix is found at $\mu_{1}=1 / 2$, or $60^{\circ}$. Nevertheless the solutions are in good agreement with numerical solutions which enforce the boundary condition of frozen foot-points on $z=0$ (e.g. Sturrock \& Barnes 1972a,b).

The derivation of scale-free solutions is readily generalised to other cases. The most important of these is perhaps the dipolar field (Aly 1994; Boily 1994, PhD Dissertation, Cambridge). Dipolar solutions with $0<p<1$ exist which discharge in the plane $z=0$ after a mean turn $N=1 / 2$.

The $p \rightarrow 0$ solution discussed herein was first worked out by one of us (Lynden-Bell 1994). Further details are in Lynden-Bell \& Boily (1994). This article was prepared with a Kluwer $\mathrm{LAT}_{\mathrm{E}} \mathrm{X}$ style file.

\section{References}

Aly, J.-J.: 1984, Astrophysical Journal, 283, 349

Aly, J.-J.: 1994, Astronomy \& Astrophysics, 288, 1012

Lynden-Bell, D.: 1994, in Cosmical Magnetism, D. Lynden-Bell (ed.), Cambridge: Institue of Astronomy, p. 16

Lynden-Bell, D. \& Boily, C.M.: 1994, MNRAS, 267, 146

Sturrock, P.A. \& Barnes, C.: 1972a,b., Astrophysical Journal, 174, 659; 176, 31 